\title{
Application of the exact exchange potential method for half metallic intermediate band alloy semiconductor
}

Cite as: J. Chem. Phys. 120, 10780 (2004); https://doi.org/10.1063/1.1737367

Submitted: 05 December 2003 . Accepted: 12 March 2004 . Published Online: 18 May 2004

J. J. Fernández, C. Tablero, and P. Wahnón

\section{ARTICLES YOU MAY BE INTERESTED IN}

Energetics of formation of $\mathrm{TiGa}_{3} \mathrm{As}_{4}$ and $\mathrm{TiGa}_{3} P_{4}$ intermediate band materials

The Journal of Chemical Physics 124, 014711 (2006); https://doi.org/10.1063/1.2140695

Analysis of metallic intermediate-band formation in photovoltaic materials

Applied Physics Letters 82, 151 (2003); https://doi.org/10.1063/1.1535744

Correlation effects and electronic properties of $\mathrm{Cr}$-substituted SZn with an intermediate band

The Journal of Chemical Physics 123, 114709 (2005); https://doi.org/10.1063/1.2034447

Lock-in Amplifiers Find out more today

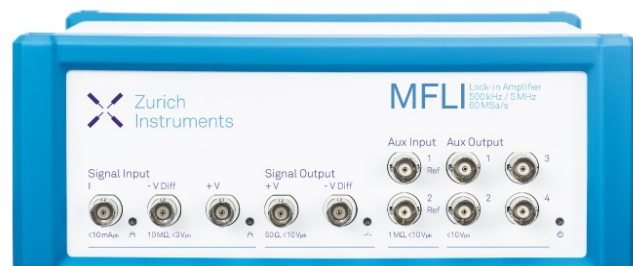

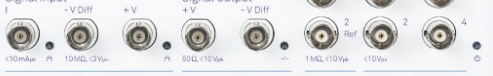

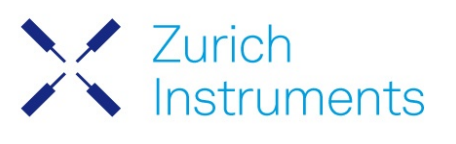




\title{
Application of the exact exchange potential method for half metallic intermediate band alloy semiconductor
}

\author{
J. J. Fernández \\ Instituto de Energía Solar and Departamento de Tecnologías Aplicadas a la Telecomunicación, ETSI \\ Telecomunicación, Universidad Politécnica de Madrid, Ciudad Universitaria s/n 28040 Madrid, Spain \\ C. Tablero ${ }^{\text {a) }}$ \\ Instituto de Energía Solar, ETSI Telecomunicación, Universidad Politécnica de Madrid, \\ Ciudad Universitaria s/n 28040 Madrid, Spain \\ P. Wahnón \\ Instituto de Energía Solar and Departamento de Tecnologías Aplicadas a la Telecomunicación, ETSI \\ Telecomunicación, Universidad Politécnica de Madrid, Ciudad Universitaria s/n 28040 Madrid, Spain
}

(Received 5 December 2003; accepted 12 March 2004)

\begin{abstract}
In this paper we present an analysis of the convergence of the band structure properties, particularly the influence on the modification of the bandgap and bandwidth values in half metallic compounds by the use of the exact exchange formalism. This formalism for general solids has been implemented using a localized basis set of numerical functions to represent the exchange density. The implementation has been carried out using a code which uses a linear combination of confined numerical pseudoatomic functions to represent the Kohn-Sham orbitals. The application of this exact exchange scheme to a half-metallic semiconductor compound, in particular to $\mathrm{Ga}_{4} \mathrm{P}_{3} \mathrm{Ti}$, a promising material in the field of high efficiency solar cells, confirms the existence of the isolated intermediate band in this compound. (C) 2004 American Institute of Physics.
\end{abstract}

[DOI: $10.1063 / 1.1737367$ ]

\section{INTRODUCTION}

The use of ab initio electronic structure calculations has become a very powerful emerging tool in many fields, especially suited to the design of new materials and pharmaceutical compounds. In spite of its great success, the KohnSham $(\mathrm{KS})^{1}$ formalism of the density functional theory $(\mathrm{DFT})^{2}$ uses approximations to the exchange-correlation part of the total energy functional. Therefore, KS methodology currently does not correctly describe some of the electronic properties of the electronic systems. Standing out among these is the treatment of the exchange term. The majority of the exchange-correlation functionals have the problem of the incomplete cancellation of the nonphysical self-interaction contained in the Coulomb energy and potential. One way of avoiding the Coulomb self-interaction problem is to treat the exchange exactly. The exchange energy is known only in terms of the one-particle KS orbitals. The explicit functional dependence of the orbitals on the electron density is unknown.

Several authors ${ }^{3,4}$ have developed new methods for approximating the exchange term using an orbital-dependent functional for the exchange potential, based on the effective potential method. ${ }^{3}$ Alternatively, because the exchange energy is known, it can be evaluated exactly, as in the exact exchange (EXX) formalism.

Many of these methods have been used in atoms and solid semiconductors. The application in solids has been car-

\footnotetext{
a) Author to whom correspondence should be addressed. Electronic mail: julio@etsit.upm.es
}

ried out mainly using a plane wave basis set. ${ }^{4}$ Recently an implementation of the EXX method has been carried out by the authors for atoms ${ }^{5}$ and solids semiconductors ${ }^{6}$ within the SIESTA code. ${ }^{7}$ This code, based on the KS methodology, uses a numerical localized basis set to represent the KS orbitals. In this EXX method an auxiliary basis set is used to represent the exchange density and solve the equation for exact-exchange potential ${ }^{5,6}$ in addition to the basis set for the KS orbitals. The auxiliary basis set used to represent the exchange density is made up of functions having the translational symmetry of the solid. Within the EXX formalism, the exchange density is built up by a combination of localized functions ${ }^{8}$

$$
\rho_{x}(\mathbf{r})=\sum_{j} C_{j} f_{j}(\mathbf{r}),
$$

where the $C_{j}$ 's are the expansion coeficients. Each one of these functions is made up of a linear combination of Slater functions centered on the atomic positions. ${ }^{6}$ The auxiliary basis set representation of the exchange density yields the correct long range $(1 / r)$ behavior of the exchange potential. As has been shown in previous papers, ${ }^{5}$ the use of the EXX method within the formalism of the linear combination of atomic orbitals leads to a stressed improvement in the description of several of the properties of some semiconductors.

Our goal in this article is to implement and apply the EXX formalism for half-metallic solids semiconductors, where the Fermi level cuts some bands. In particular, we apply this methodology in order to confirm the existence of 


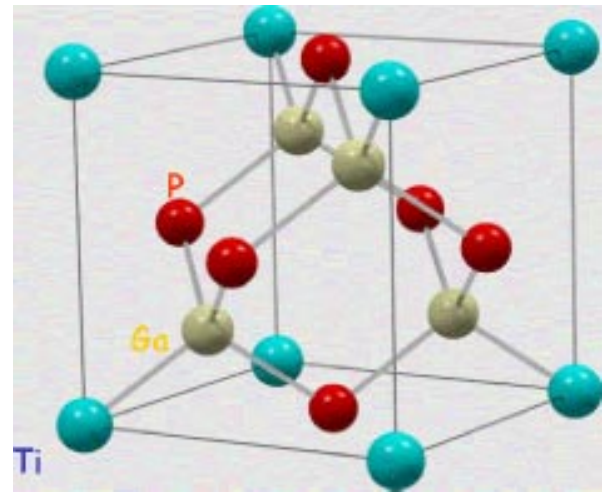

FIG. 1. The cubic crystal structure of the $\mathrm{Ga}_{4} \mathrm{P}_{3} \mathrm{Ti}$.

an isolated narrow band in a three band semiconductor, such as the half-metallic intermediate band materials already proposed $^{9}$ (MIB). This kind of material looks promising for manufacturing solar cells as a result of its ability to increase the solar cell efficiency with respect to conventional solar cells.

Luque and Marti ${ }^{10}$ have proposed the possibility of building solar cells using this new kind of material. This material (MIB) has to present a half-filled band in the middle of the bandgap. The MIB is able to absorb low energy photons to produce transitions from the valence band (VB) to the intermediate band (IB) and from the IB to the conduction band (CB). These processes improve the efficiency of the solar cell significantly. Moreover, as usual, it can also absorb photons of higher energy in order to produce transitions from the VB to the CB. These authors studied the formation and thermodynamic properties of the IB materials but they did not propose any concrete material exhibiting the desired properties.

In more recent works, ${ }^{9,11}$ and using DFT methods, Wahnón and Tablero have proposed some half-metallic materials that have an IB and have characterized its optoelectronic properties. ${ }^{12}$ One of the materials that they have proposed is the $\mathrm{Ga}_{4} \mathrm{P}_{3} \mathrm{Ti}$, an alloy formed on the basis of the $\mathrm{GaP}$ semiconductor where, in an eight atom cubic cell structure, one $\mathrm{P}$ is substituted by a Ti (Fig. 1). A detailed analysis of the IB band shows that the IB is mainly made up of $d_{x y}$, $d_{x z}$, and $d_{y z}$ orbitals of the Ti.

However, it is known that LDA overestimates the binding energy whereas GGA overestimates lattice constants, and both methods underestimate to a greater or lesser degree the bandgap energies.

Therefore, once the IB band in the $\mathrm{Ga}_{4} \mathrm{P}_{3} \mathrm{Ti}$ system has been described in terms of the usual LDA and GGA exchange correlation functionals $s^{9,11}$ it is necessary to verify whether the results obtained for the band energies and bandgaps are confirmed, especially if the presence of the isolated IB is maintained.

\section{COMPUTATIONAL DETAILS}

In this work we propose to study the $\mathrm{Ga}_{4} \mathrm{P}_{3} \mathrm{Ti}$ alloy using the recently implemented exact exchange (EXX) method that uses a basis of numerical Slater functions for the exchange density. This scheme is implemented without the restriction of full electron bands in solid semiconductors.

We will show how the use of EXX methods for studying these kinds of compounds confirms the presence of the intermediate band and the variation in the bandgaps. For this purpose we present a careful study on the convergence and properties of the band structure of the $\mathrm{Ga}_{4} \mathrm{P}_{3} \mathrm{Ti}$ material using a different auxiliary basis to represent the EXX exchange density and potential. For the GGA and EXX calculations we have used pseudopotentials generated using the GGA and EXX exchange, respectively. The same correlation term has been used for the two types of calculation.

All calculations are made using a modified version of the SIESTA code using the double- $\xi$ (DZ) or double- $\xi$ with polarization (DZP) basis sets for representing the Kohn-Sham orbitals. This code includes both the EXX potential and energies as well as the usual local density (LDA) or the generalized gradient (GGA) functionals. The GGA calculations have been made using the Perdew, Burke, and Ernzerhoff functional. $^{13}$

In these calculations, we have used the nonlocal pseudopotential approach. It has been constructed using the Troullier-Martins scheme and the Kleinmann-Bylander factorization. ${ }^{14,15}$ The reference configurations used in all the calculations were $4 s^{2} 4 p^{0} 3 d^{2}$ for $\mathrm{Ti}, 4 s^{2} 4 p^{1} 3 d^{0}$ for $\mathrm{Ga}$, and $3 s^{2} 3 p^{3} 3 d^{0}$ for P. All the generated pseudopotentials have been tested on a range of cut-off radii.

All calculations have been made using a mesh cut-off of 80 Rydbergs and a $k$-grid cut-off of $8 \AA$, including 32 $k$-points for sampling the first Brillouin zone. The cut-offs used have been tested in order to ensure the total convergence of the calculations.

The initial structure used in the GGA calculation was the eight atom $\mathrm{GaP}$ zincblende structure cell, where we have substituted a P atom with Ti (Fig. 1). In this calculation the crystalline structure parameters are obtained through a minimization of the total energy relaxing the atomic positions and the unit cell. In our case the residual forces on the atoms are required to be less than $0.001 \mathrm{eV} / \AA$ and the stress tolerance less than $0.1 \mathrm{GPa}$. The lattice parameters obtained after these relaxations, $5.88 \AA$ for DZ basis and $5.82 \AA$ for the DZP basis set, were maintained for the EXX calculations. In the EXX calculations the atoms were allowed to relax in their positions within the unit cell until the forces on the atoms were less than the aforementioned $0.001 \mathrm{eV} / \AA$ (Table I).

\section{RESULTS AND DISCUSSION}

Figure 2 shows the curves for the convergence of the total energy in the $\mathrm{Ga}_{4} \mathrm{P}_{3} \mathrm{Ti}$ system as a function of the number of basis functions used to represent the EXX exchange density. The solid and dashed lines correspond respectively to two different qualities of basis set, the DZP and DZ basis, used for representing the KS orbitals. We have plotted for each of them, eight different results obtained using from 32 to 96 auxiliary basis functions to represent the EXX exchange density. The values obtained for the converged total energies are $-869.02 \mathrm{eV}$ for the DZ basis set and $-871.91 \mathrm{eV}$ for the DZP basis set. These values are lower than the corresponding GGA values, which are -865.16 and 
TABLE I. Relaxed position of the atoms within the cubic unit cell. The positions have been obtained using the conjugate gradient method for finding the minimun energy. Values are shown in units of the relaxed lattice parameter $(a=5.92 \AA)$.

\begin{tabular}{cccc}
\hline \hline & $X$ & $Y$ & $Z$ \\
\hline $\mathrm{Ti}$ & 0.0007 & 0.0007 & 0.0007 \\
$\mathrm{P}$ & 0.0000 & 0.5001 & 0.5001 \\
$\mathrm{P}$ & 0.5001 & 0.0000 & 0.5001 \\
$\mathrm{P}$ & 0.4999 & 0.4999 & 0.0000 \\
$\mathrm{Ga}$ & 0.2607 & 0.2607 & 0.2607 \\
$\mathrm{Ga}$ & 0.2607 & 0.7392 & 0.7392 \\
$\mathrm{Ga}$ & 0.7392 & 0.2607 & 0.7293 \\
$\mathrm{Ga}$ & 0.7392 & 0.7392 & 0.2607 \\
\hline \hline
\end{tabular}

$-866.50 \mathrm{eV}$ for the DZ and the DZP basis sets, respectively. This effect in the total energy can be explained mainly in terms of the non-self-interacting character of the EXX potential. The absence of the self-interaction makes the bands more bounded and the band-energy term of the total energy more negative. At the same time the EXX exchange energy is much more negative than the GGA energy (up to $13 \%$ ): the GGA exchange energy is $-248.18 \mathrm{eV}$ and the EXX exchange energy obtained in the converged calculation is $-266.60 \mathrm{eV}$.

The behavior of the EXX exchange potential for the three different auxiliary basis sets, $\operatorname{EXX}(1), \operatorname{EXX}(2)$, and EXX(3) labeled in Fig. 2, is represented in Fig. 3 along the [111] direction in the cubic cell. The figure shows the convergence of the potential with the size of the auxiliary basis set. A DZ basis set for the Kohn-Sham potential has been used.

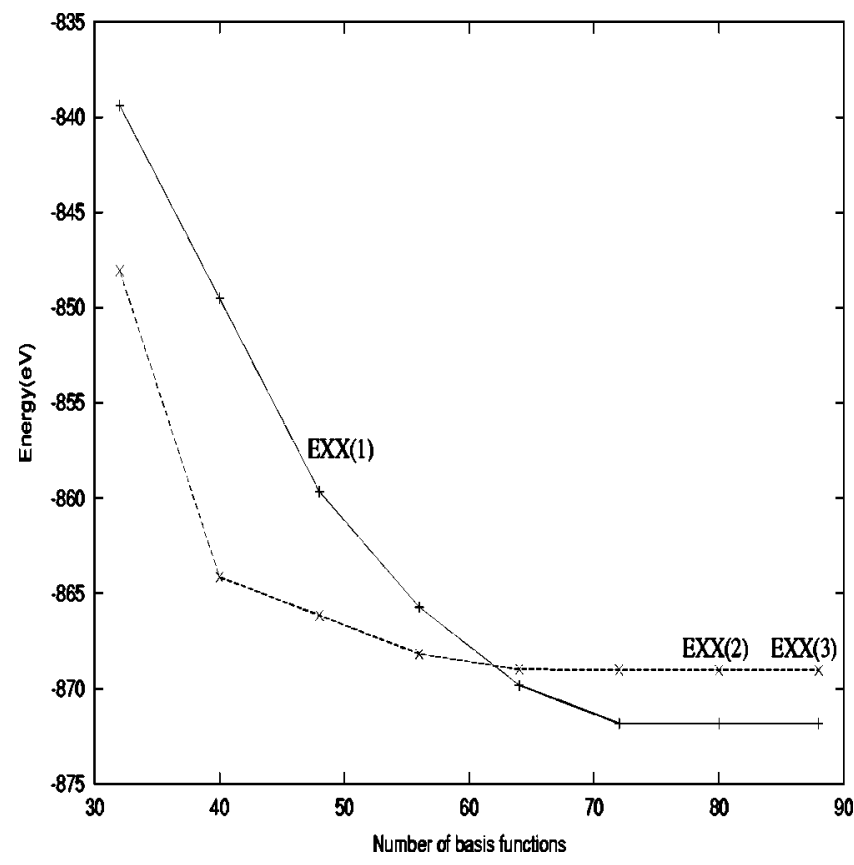

FIG. 2. Convergence of the energy $(\mathrm{eV})$ for the $\mathrm{Ga}_{4} \mathrm{P}_{3} \mathrm{Ti}$ system as a function of the number of auxiliary basis functions used to represent the exchange density. The solid line represents the convergence when the DZP basis set is used for the KS orbitals and the dashed line for the DZ basis set.

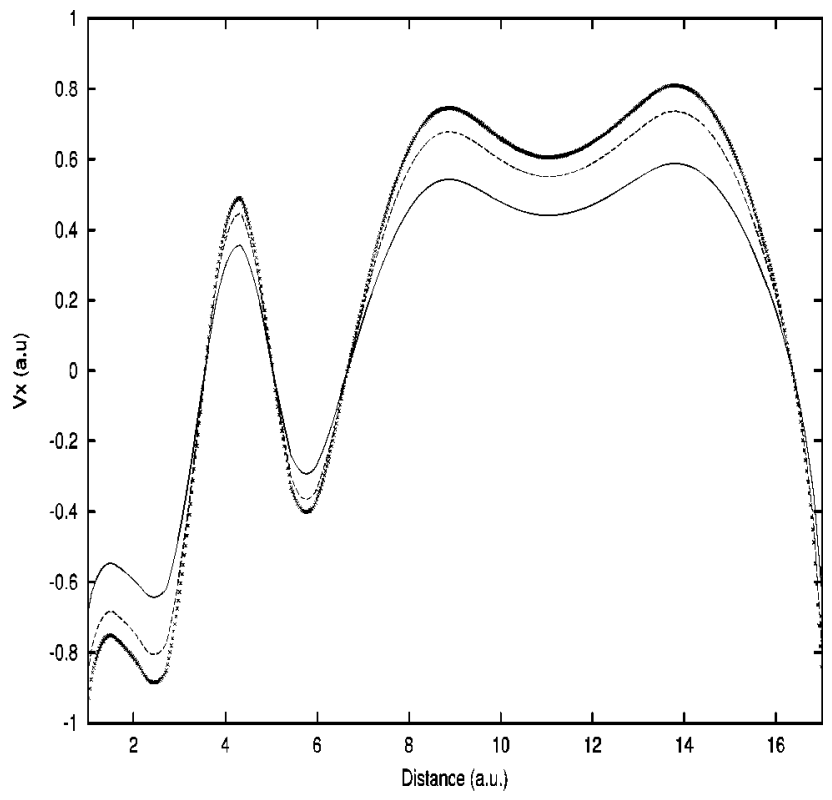

FIG. 3. EXX exchange potential, in a.u., for the $\mathrm{Ga}_{4} \mathrm{P}_{3} \mathrm{Ti}$ system using a DZ basis set, along the [111] direction of the cubic unit cell. The exchange potential is represented for the following auxiliary basis sets: (-) $\operatorname{EXX}(1),(--) \operatorname{EXX}(2),(x x x) \operatorname{EXX}(3)$

Figures 4 and 5 show the dispersion band diagrams at some points within the Brillouin zone (BZ) for the $\mathrm{Ga}_{4} \mathrm{P}_{3} \mathrm{Ti}$ system with the DZ and DZP basis sets used to represent the KS orbitals, respectively. The three different curves in each figure correspond to the two auxiliary basis sets used for expressing the exchange density and are numbered $\operatorname{EXX}(1)$ and $\operatorname{EXX}(3)$ in Fig. 2. The other curve corresponds to the GGA calculation. We have selected in the calculations these

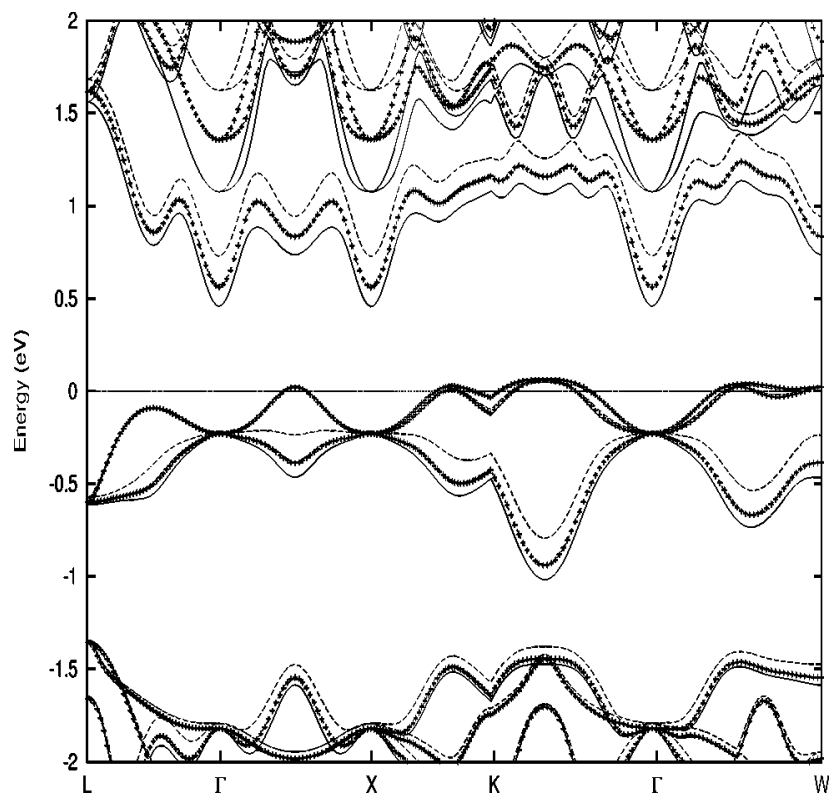

FIG. 4. Band dispersion diagrams for the $\mathrm{Ga}_{4} \mathrm{P}_{3}$ Ti system in the main directions in the Brillouin Zone. Solid lines correspond to GGA calculations while the dashed and dotted lines correspond to EXX calculations using two different auxiliary basis sets to represent the exchange density, $\operatorname{EXX}(1)$ and $\operatorname{EXX}(2)$ as labeled in Fig. 2. A DZ basis set to represent the Kohn-Sham orbitals has been used. The origin of the energies has been taken in the Fermi energy for each calculation. 


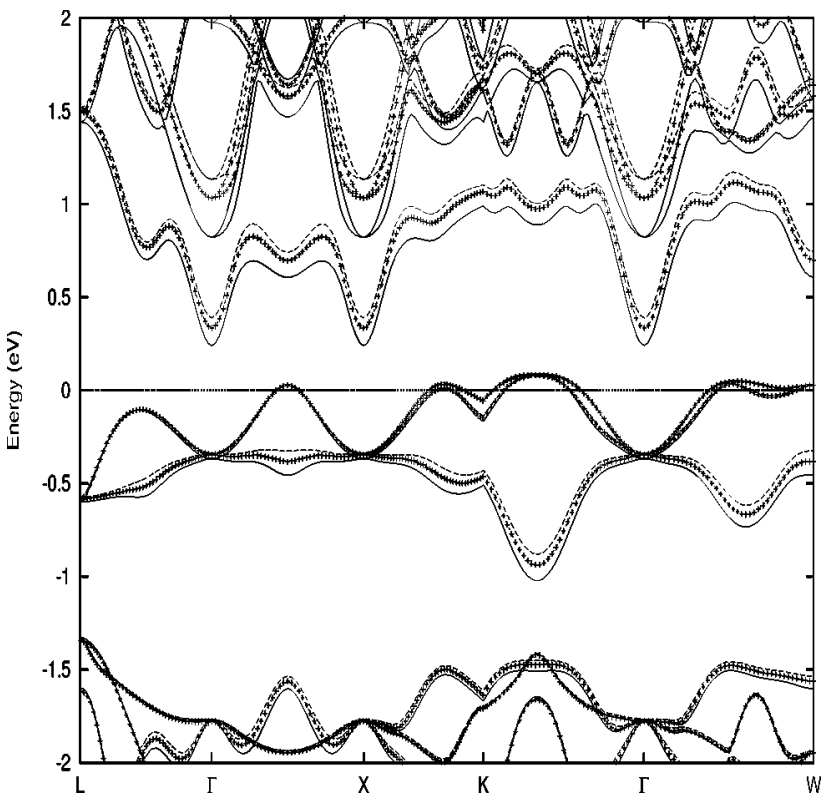

FIG. 5. The same legend as in Fig. 3 but using a DZP basis set to represent the Kohn-Sham orbitals.

two EXX-basis functions in order to appreciate properly the differences. For example, results are almost indistinguishable if we represent them for the auxiliary basis $\operatorname{EXX}(2)$ and EXX(3). In all cases the corresponding Fermi energy has been taken as the origin of the energies. Using the two aforementioned basis sets for the EXX exchange density it can be seen that the bandwidth is always smaller than in the case of the GGA calculations. The reason for this behavior is the absence of the self-interaction in the EXX method that produces a greater degree of localization in the bands, and, as a consequence, a smaller bandwidth. For the same reason, greater indirect bandgap values between the intermediate and conduction bands are satisfactorily reached for all EXX bases used.

Tables II and III show the energy values at some points in the $\mathrm{BZ}$ for the bands involved in the formation of the bandgaps and the intermediate band for the $\mathrm{Ga}_{4} \mathrm{P}_{3} \mathrm{Ti}$ using a DZ and DZP basis set respectively for the KS orbitals. One of the columns shows GGA values, and for the other three results are obtained using three different auxiliary basis set sizes to represent the exchange density. These columns are labeled $\operatorname{EXX}(1), \operatorname{EXX}(2)$, and $\operatorname{EXX}(3)$ in Fig. 2. In these tables, $E_{V}, E_{C}, E_{I}(1), E_{I}(2)$, and $E_{I}(3)$ are the energies for the valence band (VB), conduction band (CB), and three sub-bands of the IB, respectively. In Tables II and III the use of the EXX functional reduces the value of the energy in all the presented points with respect to the GGA values. This effect reflects the more local character of the EXX potential compared to the GGA exchange potential. This may be explained by the enhanced electron localization induced by the absence of self-repulsion in the EXX method. The occupied bands become more localized and energetically lower in relation to the GGA method. The comparison of the values presented in the columns labeled $\operatorname{EXX}(1), \operatorname{EXX}(2)$, and $\operatorname{EXX}(3)$ show the convergence of the energy values when a complete representation for the EXX potential is reached.
TABLE II. Energies (eV) for the conduction band $\left(E_{C}\right)$, the three subbands of the intermediate band $\left(E_{I}\right)$ numbered in increasing order of energy, and the valence band $\left(E_{V}\right)$ at some points in the $\mathrm{BZ}$ for the $\mathrm{Ga}_{4} \mathrm{P}_{3}$ Ti. The results are obtained using a DZ basis set for the KS orbitals and three different auxiliary basis sets used to represent the exchange density, labeled $\operatorname{EXX}(1)$, $\operatorname{EXX}(2)$, and $\operatorname{EXX}(3)$ as in Fig. 2.

\begin{tabular}{lllll}
\hline \hline & GGA & $\operatorname{EXX}(1)$ & $\operatorname{EXX}(2)$ & $\operatorname{EXX}(3)$ \\
\hline$L$ & & & & \\
$E_{V}$ & -5.77 & -6.05 & -6.48 & -6.48 \\
$E_{I}(1)$ & -5.01 & -5.28 & -5.70 & -5.70 \\
$E_{I}(2)$ & -5.01 & -5.28 & -5.70 & -5.70 \\
$E_{I}(3)$ & -5.01 & -5.28 & -5.70 & -5.70 \\
$E_{C}$ & -2.79 & -3.06 & -3.45 & -3.44 \\
$\Gamma$ & & & & \\
$E_{V}$ & -6.23 & -6.50 & -6.92 & -6.92 \\
$E_{I}(1)$ & -4.64 & -4.92 & -5.35 & -5.35 \\
$E_{I}(2)$ & -4.64 & -4.92 & -5.35 & -5.35 \\
$E_{I}(3)$ & -4.64 & -4.92 & -5.35 & -5.35 \\
$E_{C}$ & -3.58 & -4.09 & -4.40 & -4.39 \\
$K$ & & & & \\
$E_{V}$ & -6.05 & -6.31 & -6.70 & -6.70 \\
$E_{I}(1)$ & -4.83 & -5.09 & -5.47 & -5.46 \\
$E_{I}(2)$ & -4.53 & -4.80 & -5.23 & -5.22 \\
$E_{I}(3)$ & -4.45 & -4.73 & -5.17 & -5.17 \\
$E_{C}$ & -3.25 & -3.51 & -3.87 & -3.86 \\
$W$ & & & & \\
$E_{V}$ & -5.96 & -6.21 & -6.61 & -6.60 \\
$E_{I}(1)$ & -4.80 & -5.03 & -5.37 & -5.36 \\
$E_{I}(2)$ & -4.39 & -4.67 & -5.11 & -5.11 \\
$E_{I}(3)$ & -4.39 & -4.67 & -5.11 & -5.11 \\
$E_{C}$ & -3.58 & -3.84 & -4.19 & -4.18 \\
\hline \hline & & & &
\end{tabular}

The comparison of energy differences at different points shows that the reduction in the EXX values is nonuniform, so it does not come from a rigid transformation of the potential. Moreover, this reduction in the energy values is greater for the DZP basis set than for the DZ basis set, because of better representation of the KS orbitals in the DZP case.

Tables IV and V show the bandgaps and the width of the IB for the GGA calculation and for three different calculations using the EXX exchange functional with different sizes, labeled $\operatorname{EXX}(1), \operatorname{EXX}(2)$, and $\operatorname{EXX}(3)$ in Fig. 2. In these tables $\Delta E_{V I}$ is the bandgap between $\mathrm{VB}$ and IB, $\Delta E_{I C}$ the bandgap between IB and $\mathrm{CB}$, and $\Delta E_{I}$ is the bandwidth of the IB. As can be seen in these tables and in the dispersion band diagram displayed in Figs. 4 and 5, the values for $\Delta E_{V I}, \Delta E_{I C}$, and $\Delta E_{I}$ change considerably with respect to the GGA calculation. Moreover, these tables show that when improving the basis set representation for the EXX exchange density [increasing the size from the $\operatorname{EXX}(1)$ basis set up to the $\operatorname{EXX}(3)$ basis set], the bandgaps increase while the width of the intermediate band decreases as expected due to the non-self-interacting character of the EXX potential. When improving the basis set representation for the KS orbitals from DZ (Table IV) to DZP (Table V) the situation is similar. In this last case, there is an small reduction in the comparison of the bandgaps and the IB width values. This reduction can be explained in terms of the improvement in the electronic charge density description due to the use of a bigger basis to describe the Kohn-Sham orbitals. 
TABLE III. The same legend as in Table II but using a DZP basis set to represent the KS orbitals.

\begin{tabular}{lllll}
\hline \hline & GGA & $\operatorname{EXX}(1)$ & $\operatorname{EXX}(2)$ & $\operatorname{EXX}(3)$ \\
\hline$L$ & & & & \\
$E_{V}$ & -5.86 & -5.58 & -5.81 & -6.80 \\
$E_{I}(1)$ & -5.10 & -4.83 & -5.05 & -5.05 \\
$E_{I}(2)$ & -5.10 & -4.83 & -5.05 & -5.05 \\
$E_{I}(3)$ & -5.10 & -4.83 & -5.05 & -5.05 \\
$E_{C}$ & -3.02 & -2.77 & -2.97 & -2.97 \\
$\Gamma$ & & & & \\
$E_{V}$ & -6.29 & -6.02 & -6.24 & -6.24 \\
$E_{I}(1)$ & -4.86 & -4.60 & -4.82 & -4.82 \\
$E_{I}(2)$ & -4.86 & -4.60 & -4.82 & -4.82 \\
$E_{I}(3)$ & -4.86 & -4.60 & -4.82 & -4.82 \\
$E_{C}$ & -4.18 & -3.96 & -4.13 & -4.13 \\
$K$ & & & & \\
$E_{V}$ & -6.15 & -5.89 & -6.10 & -6.10 \\
$E_{I}(1)$ & -4.98 & -4.73 & -4.92 & -4.91 \\
$E_{I}(2)$ & -4.66 & -4.40 & -4.61 & -4.61 \\
$E_{I}(3)$ & -4.57 & -4.30 & -4.52 & -4.52 \\
$E_{C}$ & -3.45 & -3.21 & -3.40 & -3.40 \\
$W$ & & & & \\
$E_{C}$ & -6.08 & -5.82 & -6.03 & -6.03 \\
$E_{I}(1)$ & -4.90 & -4.66 & -4.84 & -4.84 \\
$E_{I}(2)$ & -4.49 & -4.21 & -4.44 & -4.44 \\
$E_{I}(3)$ & -4.49 & -4.21 & -4.44 & -4.44 \\
$E_{C}$ & -3.82 & -3.59 & -3.77 & -3.77 \\
\hline \hline & & & &
\end{tabular}

The last rows in the tables show the three previous quantities added $\left(\Delta=\Delta E_{V I}+\Delta E_{I C}+\Delta E_{I}\right)$. As can be seen, there is a small change in the total of the three quantities when the EXX and GGA values are compared. In Table V, with a DZ basis set for $\mathrm{KS}$ orbitals, the change is only $0.16 \mathrm{eV}$, which represents $8.3 \%$ of the total value. The reason for this quantity being so small is that two opposite effects are taken into account. While the gaps between the bands increase [the VB-IB bandgap grows by $0.14 \mathrm{eV}(33.3 \%)$ and the IB-CB bandgap grows by $0.18 \mathrm{eV}(36.0 \%)]$, the IB width lowers from 1.00 to $0.84(16.0 \%)$ and compensates the effect of the increase in the bandgaps. This effect can be seen in Fig. 4.

Table $\mathrm{V}$ for the DZP basis set shows, as in the DZ case, two opposite effects leading to a small change in the total energy difference between the valence and conduction bands. Thus, while the total energy difference only increases from 1.68 to $1.74 \mathrm{eV}(3.6 \%)$, the two bandgaps change by a more appreciable percentage: the bandgap from the VB to the IB increases from 0.41 to $0.46 \mathrm{eV}(12.2 \%)$ and the bandgap

TABLE IV. Bandgaps (eV) and bandwidth (eV) for the $\mathrm{Ga}_{4} \mathrm{P}_{3} \mathrm{Ti}$ system obtained using a DZ basis set to represent the Kohn-Sham orbitals. $\Delta E_{V I}$ and $\Delta E_{I C}$ are the bandgaps between VB-IB and IB-CB. $\Delta E_{I}$ represent the bandwidth of the IB.

\begin{tabular}{lcccc}
\hline \hline & GGA & $\operatorname{EXX}(1)$ & $\operatorname{EXX}(2)$ & $\operatorname{EXX}(3)$ \\
\hline$\Delta E_{V I}$ & 0.42 & 0.47 & 0.56 & 0.56 \\
$\Delta E_{I C}$ & 0.50 & 0.54 & 0.68 & 0.68 \\
$\Delta E_{I}$ & 1.00 & 0.95 & 0.84 & 0.84 \\
$\Delta^{\mathrm{a}}$ & 1.92 & 1.96 & 2.08 & 2.08 \\
\hline \hline
\end{tabular}

${ }^{\mathrm{a}} \Delta=\Delta E_{V I}+\Delta E_{I C}+\Delta E_{I}$.
TABLE V. The same legend as Table IV but using a DZP basis set.

\begin{tabular}{lcccc}
\hline \hline & GGA & $\operatorname{EXX}(1)$ & $\operatorname{EXX}(2)$ & $\operatorname{EXX}(3)$ \\
\hline$\Delta E_{V I}$ & 0.41 & 0.41 & 0.46 & 0.46 \\
$\Delta E_{I C}$ & 0.25 & 0.25 & 0.33 & 0.33 \\
$\Delta E_{I}$ & 1.02 & 1.01 & 0.96 & 0.96 \\
$\Delta^{\mathrm{a}}$ & 1.68 & 1.68 & 1.74 & 1.74 \\
\hline \hline${ }^{\mathrm{a}} \Delta=\Delta E_{V I}+\Delta E_{I C}+\Delta E_{I}$. & & &
\end{tabular}

between the IB and the $\mathrm{CB}$ increases from 0.25 to $0.33 \mathrm{eV}$ $(32.0 \%)$. There is also a reduction in the IB width from 1.02 to $0.96 \mathrm{eV}(5.9 \%)$ as the DZ basis. Figure 4 show the dispersion band diagrams that confirm these results.

\section{CONCLUSION}

We have presented a complete study of the convergence of band-structure properties of the $\mathrm{Ga}_{4} \mathrm{P}_{3}$ Ti half-metallic intermediate band system, using the implemented EXX method for calculating the exchange part of the Kohn-Sham potential and energy. The analysis of the total energy convergence for the different basis functions used shows that the system is affected in the cases studied by the use of EXX compared to GGA exchange potential. Moreover, dispersion band diagrams for the system using the EXX method compared with GGA calculations show that the bandwidth of the intermediate band is always smaller for the two qualities of the basis set used. These effects are due mainly to the nonself-interacting character of the EXX potential that makes bands more bonded as such, making the band energy term of the total energy more negative. As a final conclusion, the use of the EXX method not only confirms the results predicted previously using the DFT-LDA and DFT-GGA methods, but also the EXX calculation shows the existence of the isolated IB in the $\mathrm{Ga}_{4} \mathrm{P}_{3}$ Ti material.

\section{ACKNOWLEDGMENTS}

We are pleased to acknowledge and thank the SIESTA program authors for providing us the computational code. This work was partially supported by the European Commission Project FULLSPECTRUM (Ref. SES6-CT-2003502620).

${ }^{1}$ W. Kohn and L. J. Sham, Phys. Rev. A 140, 1133 (1965).

${ }^{2}$ P. Hohemberg and W. Kohn, Phys. Rev. B 864, 136 (1964).

${ }^{3}$ J. D. Talman and W. F. Shadwick, Phys. Rev. A 14, 36 (1976).

${ }^{4}$ M. Moukara, M. Stadele, J. A. Majewsky, P. Vogl, and A. Goerling, J. Phys.: Condens. Matter 12, 6783 (2000); M. Stadele, M. Moukara, J. A. Majewski, P. Vogl, and A. Goerling, Phys. Rev. B 59, 10031 (1999); T. Kotani and H. Akai, ibid. 54, 16502 (1996); M. Staedelle, J. A. Majewski, P. Vogl, and A. Goerling, Phys. Rev. Lett. 79, 2089 (1997).

${ }^{5}$ J. J. Fernández, C. Tablero, and P. Wahnón, Comput. Mater. Sci. 28, 274 (2003).

${ }^{6}$ J. J. Fernández, C. Tablero, and P. Wahnón, Int. J. Quantum Chem. 91, 157 (2003).

${ }^{7}$ J. M. Soler, E. Artacho, J. D. Gale, A. García, J. Junquera, P. Ordejon, and D. Sanchez-Portal, J. Phys.: Condens. Matter 14, 2745 (2002); E. Artacho, D. Sánchez-Portal, P. Ordejón, A. García, and J. M. Soler, Phys. Status Solidi B 215, 809 (1999); D. Sánchez-Portal, E. Artacho, and J. M. Soler, Int. J. Quantum Chem. 65, 453 (1997); P. Ordejón, E. Artacho, and J. M. Soler, Phys. Rev. B 53, R10441 (1996).

${ }^{8}$ A. Goerling, Phys. Rev. Lett. 83, 5459 (1999). 
${ }^{9}$ P. Wahnón and C. Tablero, Phys. Rev. B 65, 165115 (2002).

${ }^{10}$ A. Luque and A. Martí, Phys. Rev. Lett. 78, 5014 (1997).

${ }^{11}$ C. Tablero and P. Wahnón, Appl. Phys. Lett. 82(1), 151 (2003).

${ }^{12}$ C. Tablero, A. García, J. J. Fernández, P. Palacios, and P. Wahnón, Comput. Mater. Sci. 17, 58 (2003).
${ }^{13}$ J. P. Perdew, K. Burke, and M. Ernzerhof, Phys. Rev. Lett. 77, 3865 (1996); 78, 1396 (1997).

${ }^{14}$ N. Troullier and J. L. Martins, Phys. Rev. B 43, 1993 (1991).

${ }^{15}$ L. Kleinman and D. M. Bylander, Phys. Rev. Lett. 48, 1425 (1982); D. M. Bylander and L. Kleinman, Phys. Rev. B 41, 907 (1990). 